Network Working Group

R. Housley

Request for Comments: 5485

Category: Informational

\title{
Digital Signatures on Internet-Draft Documents
}

Status of This Memo

This memo provides information for the Internet community. It does not specify an Internet standard of any kind. Distribution of this memo is unlimited.

Copyright Notice

Copyright (c) 2009 IETF Trust and the persons identified as the document authors. All rights reserved.

This document is subject to BCP 78 and the IETF Trust's Legal Provisions Relating to IETF Documents in effect on the date of publication of this document (http://trustee.ietforg/license-info). Please review these documents carefully, as they describe your rights and restrictions with respect to this document.

Abstract

This document specifies the conventions for digital signatures on Internet-Drafts. The Cryptographic Message syntax (CMS) is used to create a detached signature, which is stored in a separate companion file so that no existing utilities are impacted by the addition of the digital signature. 


\section{Introduction}

This document specifies the conventions for storing a digital signature on Internet-Drafts. The Cryptographic Message Syntax (CMS) [CMS] is used to create a detached signature. The signature is stored in a separate companion file so that no existing utilities are impacted by the addition of the digital signature.

Shortly after the IETF Secretariat posts the Internet-Draft in the repository, the digital signature is generated and posted as a companion file in the same repository. The digital signature allows anyone to confirm that the contents of the Internet-Draft have not been altered since the time that the document was posted in the repository.

The signature of the IETF Secretariat is intended to provide a straightforward way for anyone to determine whether a particular file contains the document that was made available by the IETF Secretariat. The signing-time included by the IETF secretariat provides the wall-clock time; it is not intended to provide a trusted timestamp.

\subsection{Terminology}

The key words "MUST", "MUST NOT", "REQUIRED", "SHALL", "SHALL NOT", "SHOULD", "SHOULD NOT", "RECOMMENDED", "MAY", and "OPTIONAL" in this document are to be interpreted as described in RFC 2119 [STDWORDS].

\subsection{ASN.1}

The CMS uses Abstract Syntax Notation One (ASN.1) [X.680]. ASN.1 is a formal notation used for describing data protocols, regardless of the programming language used by the implementation. Encoding rules describe how the values defined in ASN.1 will be represented for transmission. The Basic Encoding Rules (BER) [X.690] are the most widely employed rule set, but they offer more than one way to represent data structures. For example, both definite-length encoding and indefinite-length encoding are supported. This flexibility is not desirable when digital signatures are used. As a result, the Distinguished Encoding Rules (DER) [X.690] were invented. DER is a subset of BER that ensures a single way to represent a given value. For example, DER always employs definite-length encoding. 


\section{Internet-Draft Signature File}

All Internet-Draft file names begin with "draft-". The next portion of the file name depends on the source of the document. For example, documents from IETF working groups usually have "ietf-" followed by the working group abbreviation, and this is followed by a string that helps people figure out the subject of the document.

All Internet-Draft file names end with a hyphen followed by a twodigit version number and a suffix. The suffix indicates the type of file. A plain text file with a suffix of ".txt" is required. Other formats may also be provided, and they employ the appropriate suffix for the file format.

The companion signature file has exactly the same file name as the Internet-Draft, except that ".p7s" is added to the end. This file name suffix conforms to the conventions in [MSG]. Here are a few example names:

Internet-Draft: draft-ietf-example-widgets-03.txt

Signature File: draft-ietf-example-widgets-03.txt.p7s

Internet-Draft: draft-ietf-example-widgets-03.ps

Signature File: draft-ietf-example-widgets-03.ps.p7s

Internet-Draft: draft-housley-internet-draft-sig-file-00.txt

Signature File: draft-housley-internet-draft-sig-file-00.txt.p7s

The IETF Secretariat will post the signature file in the repository shortly after the Internet-Draft is posted.

\subsection{Need for Canonicalization}

In general, the content of the Internet-Draft is treated like a single octet string for the generation of the digital signature. Unfortunately, the plain text file requires canonicalization to avoid signature validation problems. The primary concern is the manner in which different operating systems indicate the end of a line of text. Some systems use a single new-line character, other systems use the combination of the carriage-return character followed by a line-feed character, and other systems use fixed-length records padded with space characters. For the digital signature to validate properly, a single convention must be employed. 


\subsection{Text File Canonicalization}

The canonicalization procedure follows the conventions used for text files in the File Transfer Protocol (FTP) [FTP]. Such files must be supported by FTP implementations, so code reuse seems likely.

The canonicalization procedure converts the data from its internal character representation to the standard 8-bit NVT-ASCII representation (see TELNET [TELNET]). In accordance with the NVT standard, the <CRLF> sequence MUST be used to denote the end of a line of text. Using the standard NVT-ASCII representation means that data MUST be interpreted as 8-bit bytes.

Trailing space characters MUST NOT appear on a line of text. That is, the space character must not be followed by the <CRLF> sequence. Thus, a blank line is represented solely by the <CRLF> sequence.

The form-feed nonprintable character $(0 \times 0 \mathrm{C})$ is expected in InternetDrafts. Other nonprintable characters, such as tab and backspace, are not expected, but they do occur. For robustness, any nonprintable or non-ASCII characters (ones outside the range 0x20 to 0x7E) MUST NOT be changed in any way not covered by the rules for end-of-line handling in the previous paragraph.

Trailing blank lines MUST NOT appear at the end of the file. That is, the file must not end with multiple consecutive <CRLF> sequences.

Any end-of-file marker used by an operating system is not considered to be part of the file content. When present, such end-of-file markers MUST NOT be processed by the digital signature algorithm.

Note: This text file canonicalization procedure is consistent with the ASCII NVT definition offered in Appendix B of RFC 5198 [UFNI].

\subsection{XML File Canonicalization}

In accordance with the guidance of the World Wide Web Consortium (W3C) in Section 2.11 of [R20060816], a <LF> character MUST be used to denote the end of a line of text within an XML file. Any twocharacter $\langle\mathrm{CRLF}>$ sequence and any $\langle\mathrm{CR}>$ that is not followed by $<\mathrm{LF}>$ are to be translated to a single $\langle\mathrm{LF}\rangle$ character.

\subsection{Canonicalization of other File Formats}

No canonicalization is needed for file formats currently used for Internet-Drafts other than plain text files and XML files. Other file formats are treated as a simple sequence of octets by the digital signature algorithm. 
3. CMS Profile

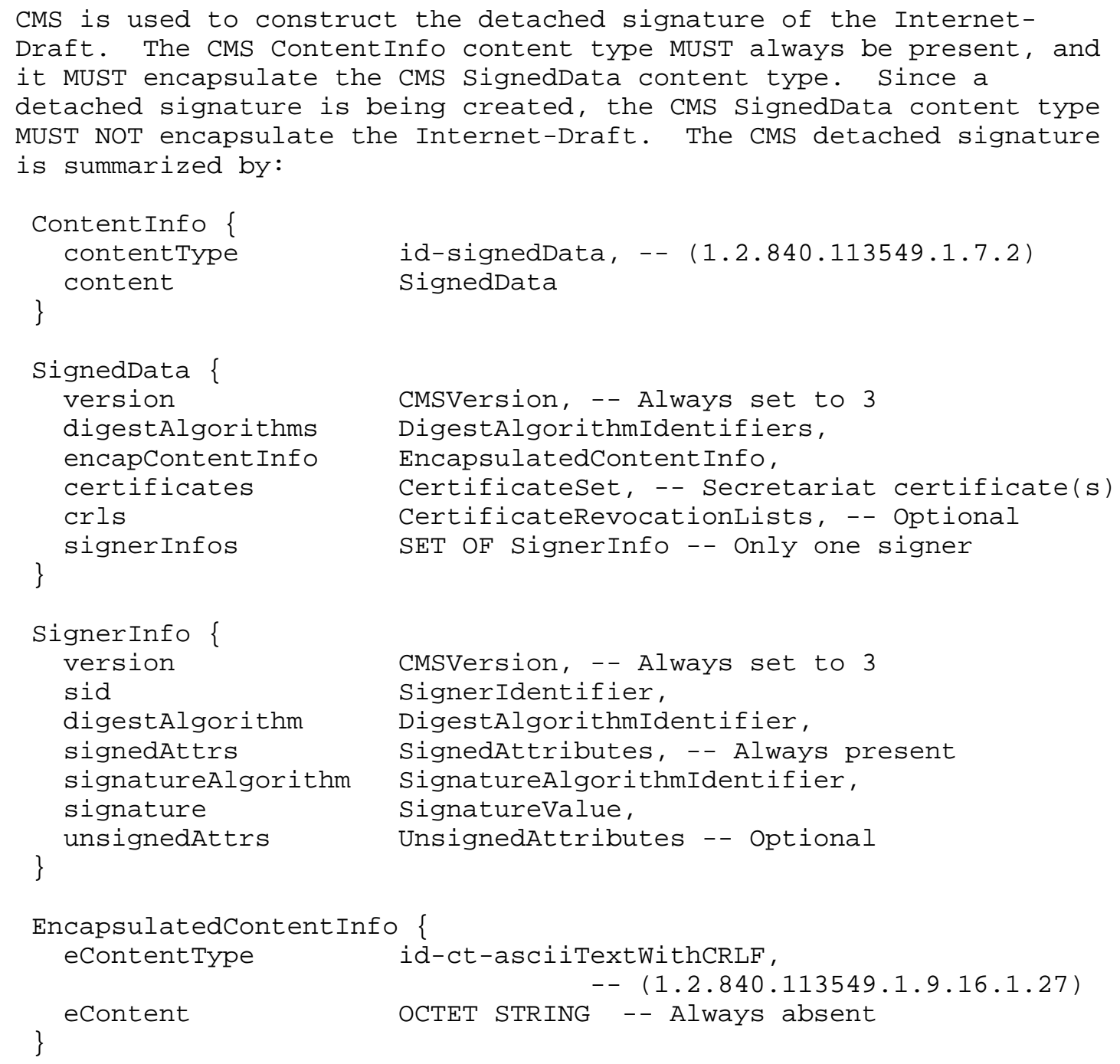

eContentType id-ct-ascitiTextWithCRLF, -- (1.2.840.113549.1.9.16.1.27) 


\subsection{Contentinfo}

The CMS requires the outer-most encapsulation to be ContentInfo [CMS]. The fields of ContentInfo are used as follows:

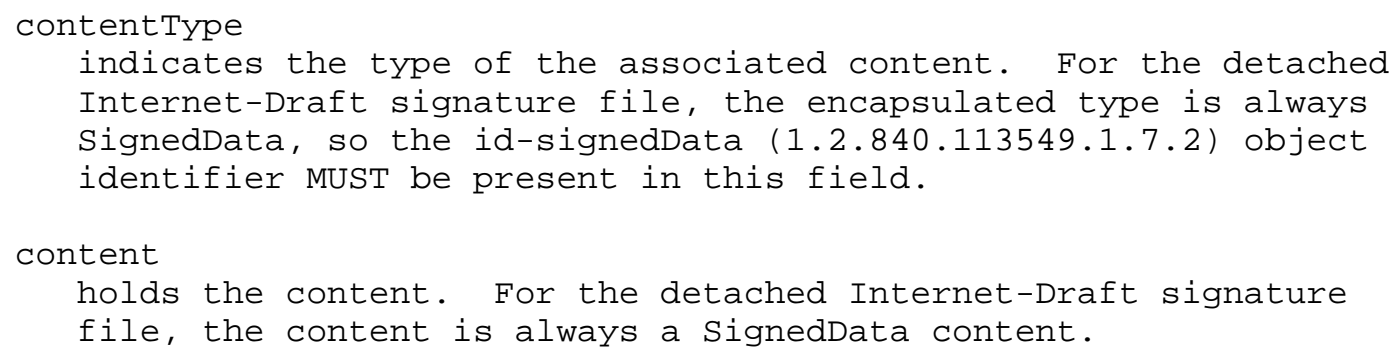

\subsection{SignedData}

The SignedData content type [CMS] contains the signature of the Internet-Draft and information to aid in the validation of that signature. The fields of SignedData are used as follows:

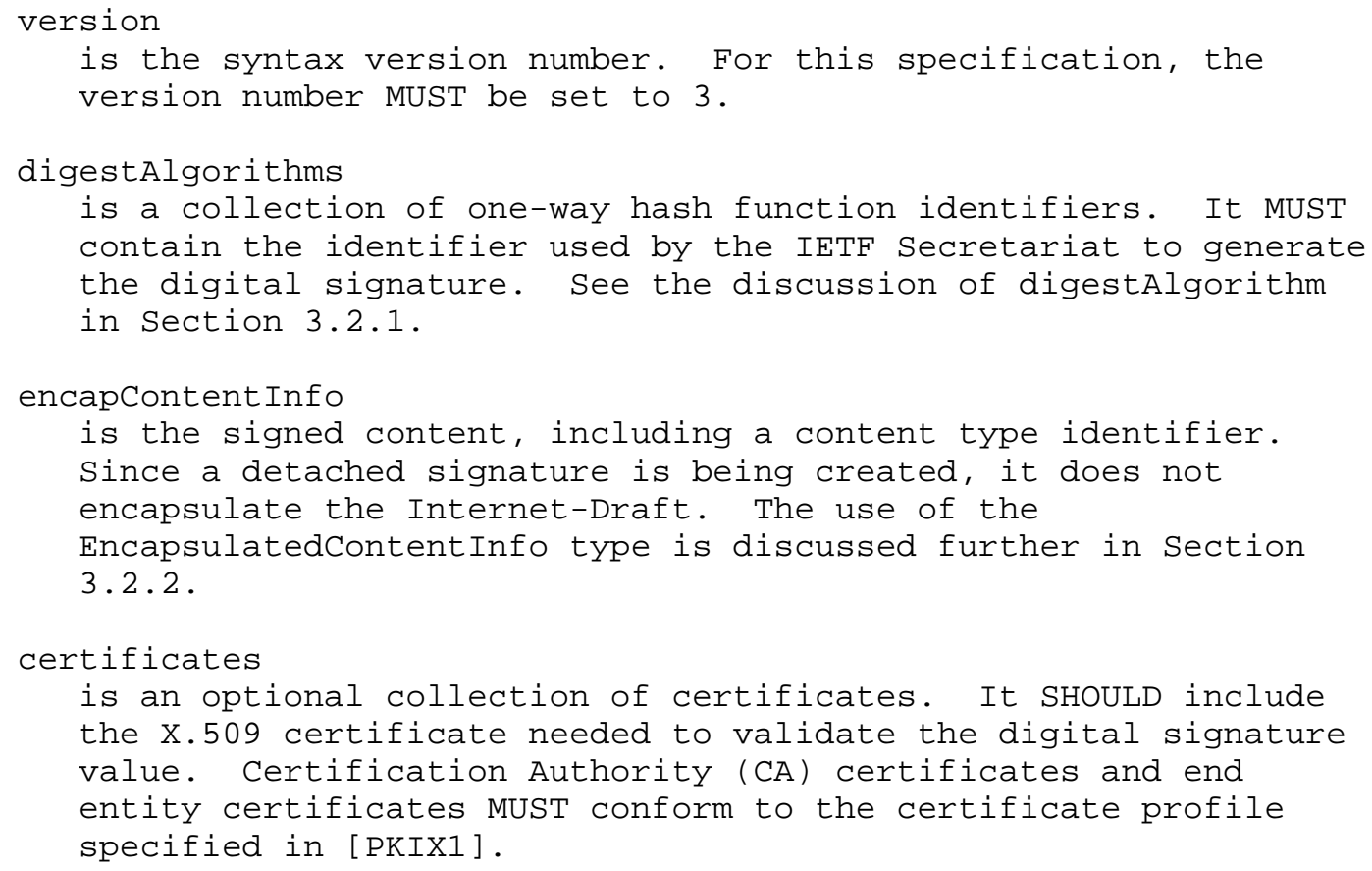




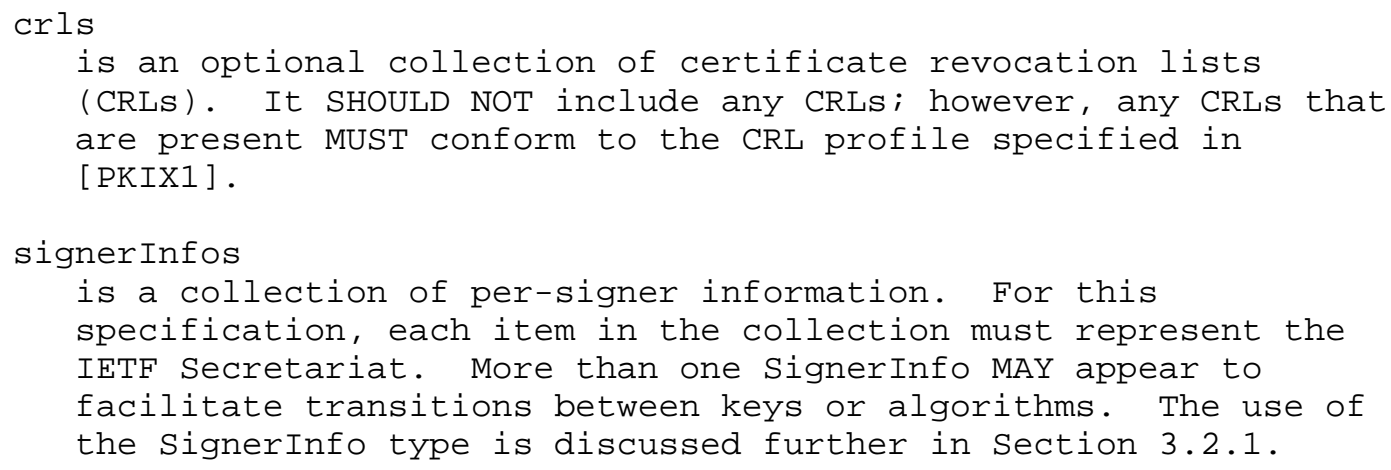

\subsubsection{SignerInfo}

The IETF Secretariat is represented in the SignerInfo type. The fields of SignerInfo are used as follows:

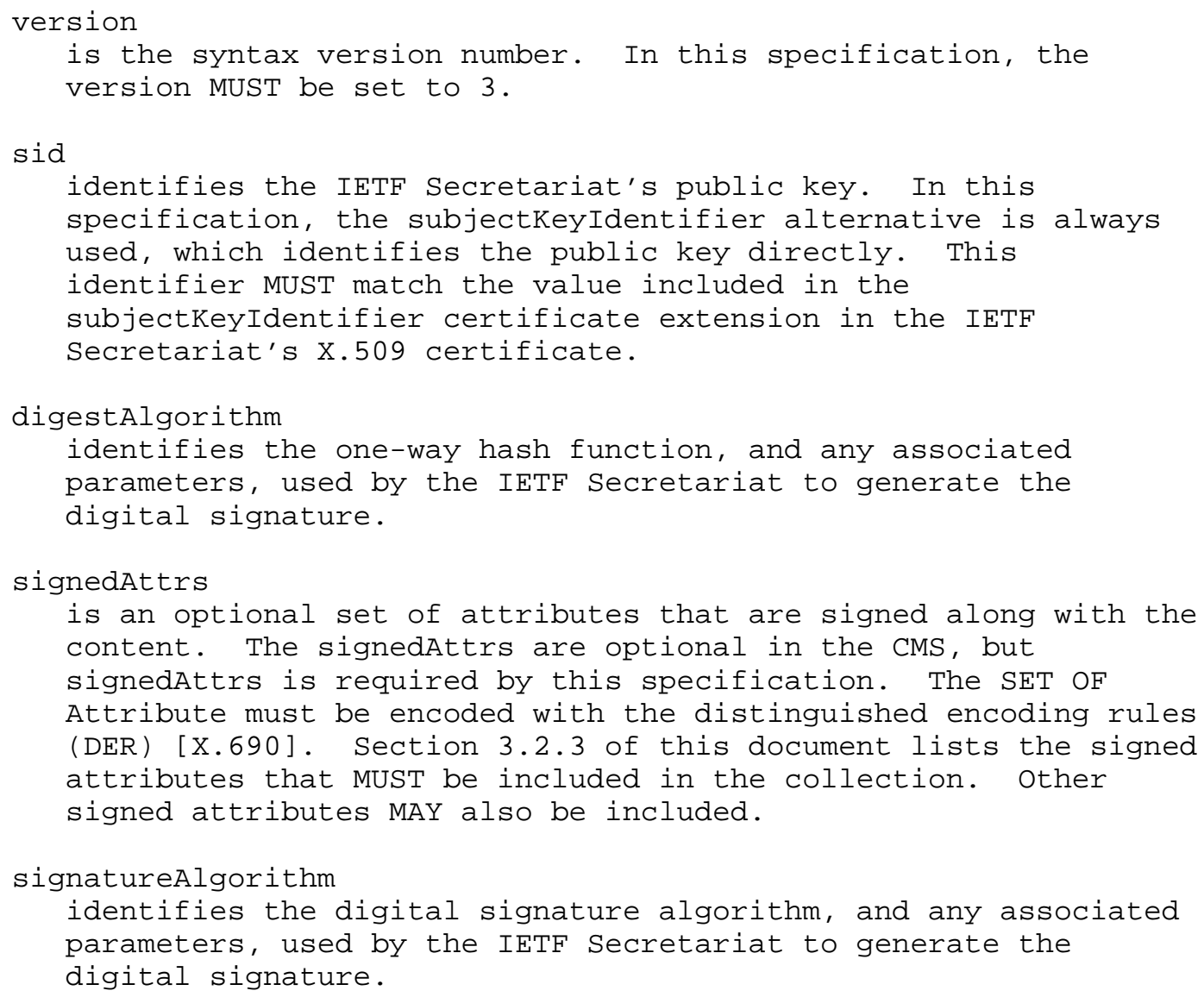




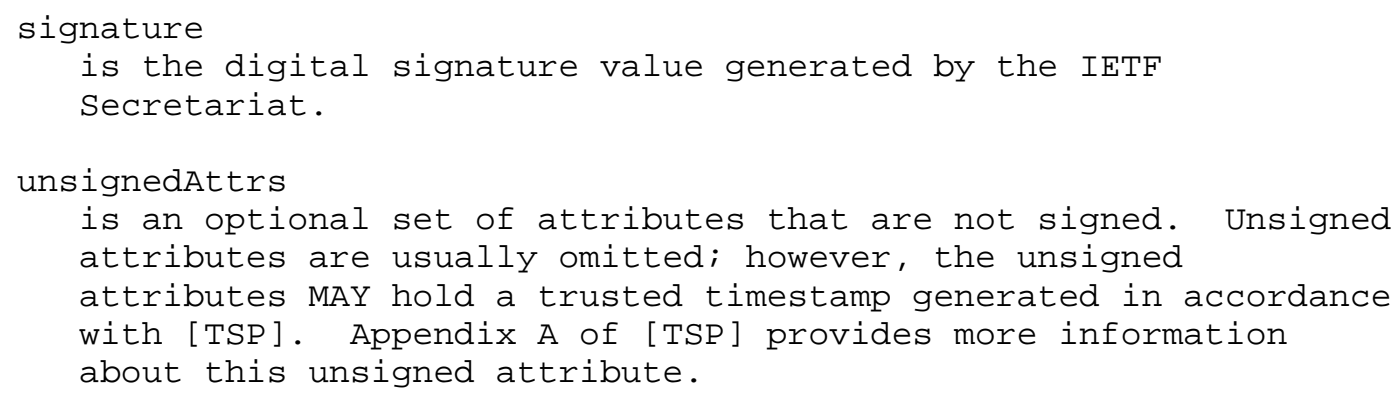

\subsubsection{EncapsulatedContentInfo}

The EncapsulatedContentInfo structure contains a content type identifier. Since a detached signature is being created, it does not encapsulate the Internet-Draft. The fields of EncapsulatedContentInfo are used as follows:

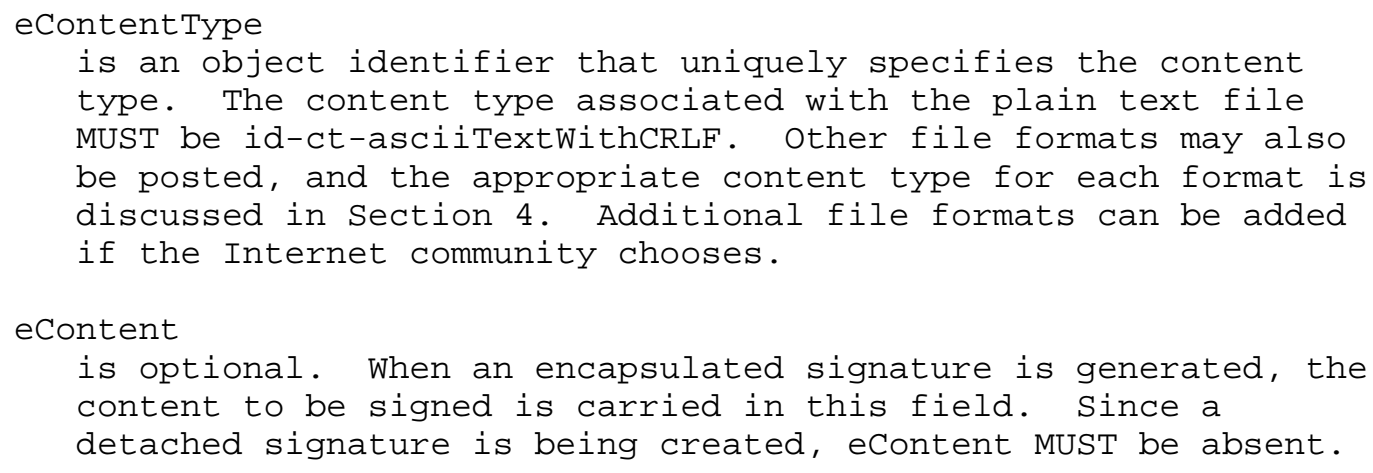

\subsubsection{Signed Attributes}

The IETF Secretariat MUST digitally sign a collection of attributes along with the Internet-Draft. Each attribute in the collection MUST be DER-encoded. The syntax for attributes is defined in [X.501], and the X.500 Directory provides a rich attribute syntax. A very simple subset of this syntax is used extensively in [CMS], where ATTRIBUTE.\&Type and ATTRIBUTE.\&id are the only parts of the ATTRIBUTE class that are employed.

Each of the attributes used with this CMS profile has a single attribute value. Even though the syntax is defined as a SET OF AttributeValue, there MUST be exactly one instance of AttributeValue present. 
The SignedAttributes syntax within signerInfo is defined as a SET OF Attribute. The SignedAttributes MUST include only one instance of any particular attribute.

The IETF Secretariat MUST include the content-type, message-digest, and signing-time attributes. The IETF Secretariat MAY also include the binary-signing-time signed attribute as well as any other attribute that is deemed appropriate. The intent is to allow additional signed attributes to be included if a future need is identified. This does not cause an interoperability concern because unrecognized signed attributes are ignored at verification.

\subsubsection{Content-Type Attribute}

A content-type attribute is required to contain the same object identifier as the content type contained in the EncapsulatedContentInfo. The appropriate content type for each format is discussed in section 4. The IETF Secretariat MUST include a content-type attribute containing the appropriate content type. Section 11.1 of [CMS] defines the content-type attribute.

\subsubsection{Message-Digest Attribute}

The IETF Secretariat MUST include a message-digest attribute, having as its value the output of a one-way hash function computed on the Internet-Draft that is being signed. Section 11.2 of [CMS] defines the message-digest attribute.

\subsubsection{Signing-Time Attribute}

The IETF Secretariat MUST include a signing-time attribute, specifying the time, based on the local system clock, at which the digital signature was applied to the Internet-Draft. Since the IETF Secretariat may choose to perform signatures in batches, the signingtime may be several hours or days after the time that the InternetDraft was actually posted. Section 11.3 of [CMS] defines the content-type attribute.

\subsubsection{Binary-Signing-Time Attribute}

The IETF Secretariat MAY include a binary-signing-time attribute, specifying the time at which the digital signature was applied to the Internet-Draft. If present, the time that is represented MUST match the time represented in the signing-time attribute. The binarysigning-time attribute is defined in [BinTime]. 


\subsubsection{Unsigned Attributes}

Unsigned attributes are usually omitted. However, an unsigned attribute MAY hold a trusted timestamp generated in accordance with [TSP]. The idea is to timestamp the IETF secretariat digital signature to prove that it was created before a given time. If the IETF Secretariat's certificate is revoked the timestamp allows a verifier to know whether the signature was created before or after the revocation date. Appendix A of [TSP] defines the signature timestamp attribute that can be used to timestamp a digital signature.

4. Content Types

This section lists the content types that are used in this specification. The econtentType field as described in section 3.2.2 contains a content type identifier, and the same value appears in the content-type attribute as described in section 3.2.3.1.

The following table lists the file formats and the associated content type.

$\begin{array}{ll}\text { File Format } & \text { Content Type } \\ \text {--------- } & ---------- \\ \text { Plain text } & \text { id-ct-asciiTextWithCRLF } \\ \text { Extensible Markup Language (XML) } & \text { id-ct-xml } \\ \text { Portable Document Format (PDF) } & \text { id-ct-pdf } \\ \text { PostScript } & \text { id-ct-postscript }\end{array}$

The object identifiers associated with the content types listed in the above table are:

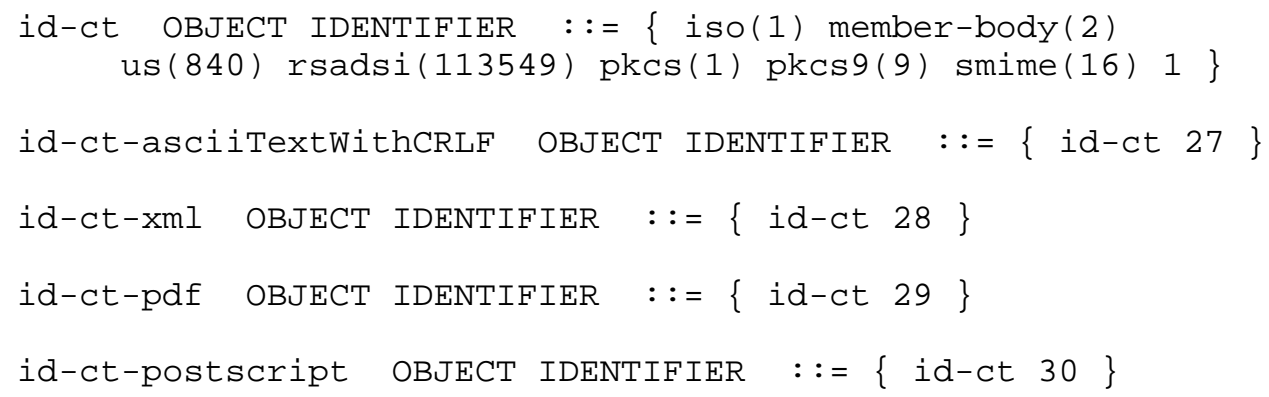

5. Security Considerations

The IETF Secretariat MUST protect its private key. The use of a hardware security module (HSM) is strongly RECOMMENDED because compromise of the IETF Secretariat's private key permits masquerade. 
The IETF Secretariat currently maintains servers at a primary location and a backup location. This configuration requires two HSMs, one at each location. However, the two HSMs do not need to use the same signing key. Each HSM can have a different signing key, as long as each one has their own certificate.

The generation of a public/private key pair for signature operations relies on random number generation. The use of an inadequate pseudorandom number generator (PRNG) can result in little or no security. An attacker may find it much easier to reproduce the PRNG environment that produced the key pair, searching the resulting small set of possibilities, than to brute-force search the whole private key space. The generation of quality random numbers is difficult, but [RANDOM] offers important guidance in this area.

The IETF Secretariat should be aware that cryptographic algorithms become weaker with time. As new cryptanalysis techniques are developed and computing performance improves, the work factor to break a particular digital signature algorithm or one-way hash function will be reduced. Therefore, it sHould be possible to migrate these algorithms. That is, the IETF Secretariat SHOULD be prepared for the supported algorithms to change over time.

The IETF Secretariat must take care to use the correct time in signing-time and binary-signing-time attributes. The inclusion of a date within the Internet-Draft by the authors that is shortly before the signing time attributes supplied by the IETF secretariat provides confidence about the date that the Internet-Draft was posted to the repository. However, the IETF Secretariat may choose to perform signatures in batches, and the signing-time may be several hours or days after the time that the Internet-Draft was actually posted.

As stated above, the IETF Secretariat may choose to sign InternetDrafts in batches. This allows a single HSM to be used if multiple servers are located in one geographic location, and it allows the HSM to be off-line except when signatures are being generated. Further, this allows the IETF secretariat to include manual steps, such as entering an HSM passphrase or inserting a smartcard, as part of the signing procedure to improve operations security.

6. Deployment and Operational Considerations

The private key used to generate the IETF Secretariat signature ought to be stored in an HSM to provide protection from unauthorized disclosure. While the HSM will be operated by the IETF Secretariat, it ought to be owned by the IETF Trust. Accordingly, the Trustees of the IETF Trust will designate an appropriate certification authority 
to issue a certificate to the IETF Secretariat, and they will approve any procedures used by the IETF secretariat for signing documents consistent with this specification.

\section{Design Rationale}

A detached signature is used for all file formats. Some file formats, such as PDF and XML, have file-format-specific ways of handling digital signatures. These file-format-specific approaches are not used for two reasons. First, a single way to sign InternetDrafts will ease implementation by the IETF Secretariat. Second, if the author includes a signature using one of these file-formatspecific approaches, the IETF Secretariat signature does not harm it in any way.

File names are the means linking the detached signature to the signed document. A CMS signed attribute could have been specified to include another form of linkage, and this could be added in the future. At this point in time, it is important to support signature validation of expired Internet-Drafts that are obtained from non-IETF repositories. Therefore, the appropriate value for such a signed attribute is unclear. This specification allows an Internet-Draft and companion signature file to be stored anywhere without hindering signature validation.

8. Acknowledgments

The idea for the Internet-Draft signature file came from a discussion with Scott Bradner at IETF 69 in Chicago. Many helpful suggestions came from Jim Schaad, Pasi Eronen, and Chris Newman.

9. References

\subsection{Normative References}

[CMS] Housley, R., "Cryptographic Message Syntax (CMS) ", RFC 3852, July 2004 .

[PKIX1] Cooper, D., Santesson, S., Farrell, S., Boeyen, S., Housley, R., and W. Polk, "Internet X.509 Public Key Infrastructure Certificate and Certificate Revocation List (CRL) Profile", RFC 5280, May 2008.

[STDWORDS] Bradner, S., "Key words for use in RFCs to Indicate Requirement Levels", BCP 14, RFC 2119, March 1997. 
[X.680] ITU-T Recommendation X.680: ISO/IEC 8824-1:2002, Information technology - Abstract Syntax Notation One (ASN.1): Specification of basic notation, 2002.

[X.690] ITU-T Recommendation X.690: ISO/IEC 8825-1:2002, Information technology - ASN.1 encoding rules: Specification of Basic Encoding Rules (BER), Canonical Encoding Rules (CER) and Distinguished Encoding Rules $(\mathrm{DER}), 2002$.

\subsection{Informative References}

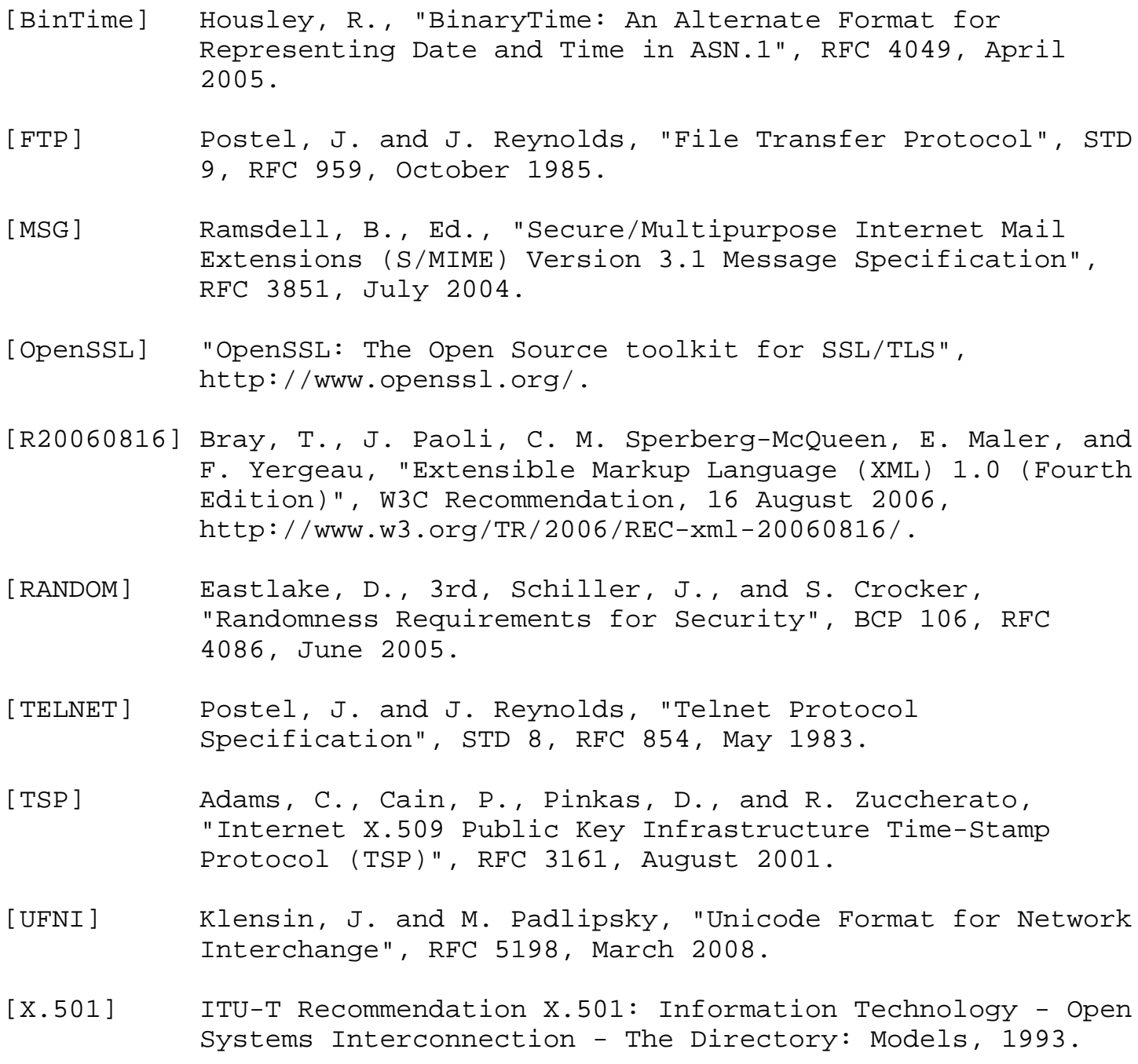

[R20060816] Bray, T., J. Paoli, C. M. Sperberg-McQueen, E. Maler, and F. Yergeau, "Extensible Markup Language (XML) 1.0 (Fourth Edition)", W3C Recommendation, 16 August 2006, http: //www.w3.org/TR/2006/REC-xml-20060816/.

[RANDOM] Eastlake, D., 3rd, Schiller, J., and S. Crocker, "Randomness Requirements for Security", BCP 106, RFC 4086, June 2005.

[TELNET] Postel, J. and J. Reynolds, "Telnet Protocol Specification", STD 8, RFC 854, May 1983.

[TSP] Adams, C., Cain, P., Pinkas, D., and R. Zuccherato, "Internet X.509 Public Key Infrastructure Time-Stamp Protocol (TSP)", RFC 3161, August 2001.

[UFNI] Klensin, J. and M. Padlipsky, "Unicode Format for Network Interchange", RFC 5198, March 2008.

[X.501] ITU-T Recommendation X.501: Information Technology - Open systems Interconnection - The Directory: Models, 1993. 
Appendix: A

OpenSSL 0.9.9 [OpenSSL] includes an implementation of CMS. The following command line can be used to verify an Internet-Draft signature:

openssl cms -verify -CAfile <cert-file> -content <internet-draft> / -inform DER -in <p7s-file> -out /dev/null

The arguments need to be provided as follows:

$<$ cert-file>

the name of the file containing the trust anchor, which is

typically the self-signed certificate of the certification

authority that issued a certificate to the IETF Secretariat.

<internet-draft>

the name of the file containing the Internet-Draft after canonicalization.

<p7s-file

the name of the file containing the detached signature that was generated in accordance with this specification.

Author's Address

Russell Housley

Vigil Security, LLC

918 Spring Knoll Drive

Herndon, VA 20170

USA

EMail: housley@vigilsec.com 